\title{
Research and Validation of Lightning Location Based on Particle Swarm Optimization Algorithm
}

\author{
Xiaohong Guo ${ }^{1, \mathrm{a}}$, Yongli Zhu ${ }^{1, \mathrm{~b}}$, Hua Chen ${ }^{2}$, Lei Zhao ${ }^{1, \mathrm{c}}$, Xu Chen $^{1}$ and \\ Yanfeng $\mathrm{Gao}^{1}$ \\ ${ }^{1}$ North China Electric Power University, Baoding 071003, China \\ ${ }^{2}$ Jibei Electric Power Maintenance Company, Beijing 102488, China \\ aguoxiaohong_hd@126.com, byonglipw@163.com, 'chhaoleizozelar@163.com
}

Keywords: LLS, particle swarm optimization, PSO.

\begin{abstract}
In the lightning monitoring systems, positioning calculation is directly related to the results of the detection accuracy. In this paper, the concept of the particle swarm optimization (PSO) algorithm for lightning location estimation was brought in. The PSO overcome the disadvantages of iterative method, such as the difficulty in finding initial and going to diverge. The numerical simulation results show that: the algorithm can obtained lightning point steadily and accurately, and converge quickly. Therefore, the PSO algorithm on lightning location is feasible.
\end{abstract}

\section{Introduction}

In the lightning monitoring systems, positioning calculation is directly related to the results of the detection accuracy [1]. The algorithms of lightning location generally use Taylor series and least squares iterative algorithm, which have the shortcomings such as difficult to determine the initial value or easy to diverge. Based on the above, this paper introduces the PSO into the lightning location, and make the numerical simulation and validation.

\section{Lightning location Based on Particle Swarm Optimization Algorithm}

Brief review of the PSO theory and algorithm. The PSO is an effective global optimization algorithms. The basic idea of the PSO is to achieve searching for optimal solutions in complex spatial through collaboration and information sharing among groups of individuals. The PSO adopts "Speed-Shift" model for action [2]. In each generation population, the particles will track the two extremes: one is the optimal solution the particle itself found so far, namely its extreme [3]; the other is the optimal solution the whole population found so far, namely the global extremum [4]. These two extremes continuously adjust the position of the particle which can be found the optimal solution within a few iterations.

PSO can be described as: Let PSO search in an n-dimensional space, the population consists of $\mathrm{N}$ particles $\mathrm{X}=\left\{X_{1}, X_{2}, \ldots, X_{N}\right\}$. Each particle location $\mathrm{X}_{i}=\left\{x_{i 1}, x_{i 2}, \ldots, x_{i n}\right\}$ represents a solution of the problem. The particles search for the new solutions by constantly adjusting their positions. Particles by continuously adjust their position $\left(x_{i d}\right)$ to search for a new solution. Each particle can remember their optimal solutions they have searched for, and the best position $\left(p_{g}\right)$ the entire particle swarm have went by, which is also the optimal solution searched currently, denoted $p_{g}$. In addition, each particle has a velocity, denoted by $\mathrm{V}_{i}=\left\{v_{i 1}, v_{i 2}, \ldots v_{i n}\right\}$, while the latter two are found, each particle will update their own pace according to Eq. 1.

$$
\begin{aligned}
& v_{i}(t+1)=w v_{\mathrm{i}}(t)+c_{1} R_{m 1}\left(p_{i}-x_{i}(t)+c_{2} R_{m 2}\left(p_{g}-x_{i}(t)\right.\right. \\
& x_{i}(t+1)=x_{i}(t)+v_{i}(t+1)
\end{aligned}
$$

Where $v_{i d}(t+1)$ represents the $\mathrm{i}$-th particle velocity at $\mathrm{t}+1$ iterations. $w$ is the inertia weight, and it can reduce the flight speed of the particle and prevent search divergence; $c_{1}, c_{2}$ is 
acceleration constant, generally take $c_{1}=c_{2}=2 ; R_{m 1}, R_{m 2}$ for $\mathrm{n} \times \mathrm{n}$-dimensional diagonal matrix, the diagonal elements are random number between [0,1]. In addition, the speed of the particles will not be too large, and you can set the speed $\operatorname{limit}\left(v_{\max }\right)$. That is, in Eq. 1 when $v_{i}(t+1)>v_{\max }, v_{i}(t+1)=v_{\max }$; when $v_{i}(t+1)<v_{\max }, v_{i}(t+1)=-v_{\max }$.

Inertia weight $w$ is given by Eq. 3:

$$
\mathrm{w}=\left(\mathrm{w}_{\text {start }}-\mathrm{w}_{\text {end }}\right) \times \frac{(\text { MaxDT-iter })}{\text { MaxDT }}+w_{\text {end }}
$$

Where MaxDT is the maximum number of iterations; Iter is the current iteration number; $\mathrm{w}_{\text {start }}, \mathrm{w}_{\text {end }}$ were initial inertia weight and termination inertia weight, $\mathrm{w}_{\text {start }}=0.9, \mathrm{w}_{\text {end }}=0.4$. PSO implementation steps are as follows:

(1) Initialized. Set various parameters PSO algorithm have been involved.

(2) Calculate the fitness of each particle (fitness). Store the best place Pbest of each particle and fitness. Choose the best fitness position of the particle from the population as Gbest of populations;

(3) Update state of the particles according to Eq. 1 and Eq.2;

(4) If the current situation reaches the maximum number of iterations or final result is less than the convergence precision, stop the iterative and output the optimal solution. Otherwise go to step (2).

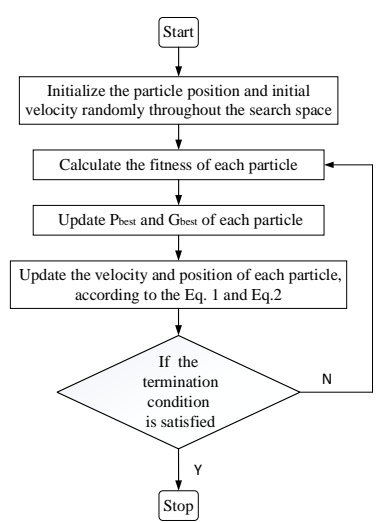

Fig.1 The flow chart of PSO algorithm

Adopt the PSO to achieve LLS. We usually use electromagnetic waves to locate, such as Magnetic Directional Orientation (MDF), Time Of Arrival (TOA) and integrated lightning location ("MDF + TOA").

Ground flash's coordinates are $\mathrm{X}_{i}\left(x_{i}, y_{i}\right)$ and occurrence time is t. Plane position coordinate of detection station is $S_{m}\left(x_{m}, y_{m}\right)(\mathrm{m}=1,2, \ldots, \mathrm{n})$. The electromagnetic wave propagation velocity is $c=299792458 \mathrm{~m} / \mathrm{s}$ [5]. Arrival time $\mathrm{t}_{i}$ and arrival direction $\varphi_{i}$ of ground flash signals are observed in the probe station:

$$
\begin{aligned}
\mathrm{t}_{i} & =t+\frac{d_{i}}{c}+\varepsilon_{i} \\
\varphi_{i} & =\arctan \frac{y_{i}-y}{x_{i}-x}+e_{i}
\end{aligned}
$$

Among them, the distance between lightning position and the probe station is $d_{i}=\sqrt{\left(x_{i}-x\right)^{2}+\left(y_{i}-y\right)^{2}} ; \varepsilon_{i}$ and $e_{i}$ are random errors of time and direction measurement. Due to the randomness of the measurement, we can assume that they are respectively normal distribution of zero mean, variances are $\sigma_{T}{ }^{2}, \sigma_{a}{ }^{2}$, in practical application can set $\sigma_{T}=1 \mu \mathrm{s}, \sigma_{a}=1^{\circ}$.

When set the lightning position and time as the solving target, the PSO need search in the xyt-three-dimensional space. But in order to reduce the amount of calculation, the PSO search in the xy-two-dimensional space when using the TOA. The corresponding fitness function is shown in Eq.6. 


$$
\mathrm{F}\left(X_{m}\right)=\frac{1}{\sigma_{T}^{2}} \sum_{i=1}^{n}\left(t_{i}-\frac{d_{i}\left(X_{m}\right)}{c}-t_{0}\right)^{2}
$$

In the formula, $d_{i}\left(X_{m}\right)$ is the distance between particle position and the i-th probe station. $t_{0}$ is the average time that each lightning detection stations speculate the time of occurrence.

$$
t_{0}=\frac{1}{n} \sum_{i=1}^{n}\left(t_{i}-\frac{d_{i}\left(X_{m}\right)}{c}\right)
$$

When adopt the lightning location combined with Directional Positioning and Arrival Time Positioning. The corresponding fitness function is shown as Eq.8.

$$
\mathrm{F}\left(X_{m}\right)=\frac{1}{\sigma_{T}^{2}} \sum_{i=1}^{n}\left(t_{i}-\frac{d_{i}\left(X_{m}\right)}{c}-t_{0}\right)^{2}+\frac{1}{\sigma_{a}^{2}} \sum_{i=1}^{n}\left(\varphi_{i}-\arctan \frac{y_{m}-y_{i}}{x_{m}-x_{i}}\right)^{2}
$$

Numerical simulation and verification based on PSO LLS. Probe stations' locations are $\mathrm{S}_{1}[0$, $0], S_{2}[0,100], S_{3}[-86.6,-50], S_{4}[86.6,-50]$ (units are km). May wish to set the lightning position is $X[25,25](\mathrm{km})$, and time of occurrence is $t=0$ s.Assuming the time and direction are free of measurement error, that is $\boldsymbol{\varepsilon}_{\boldsymbol{i}}=\mathbf{0}, \boldsymbol{e}_{\boldsymbol{i}}=\mathbf{0}$.

Eq. (2-6) as a function of particle adapt PSO algorithm called TOA-PSO algorithm. Eq.8 as a function of particle adapt PSO algorithm called TOA/DF algorithm.

TOA-PSO algorithm. Apparent from the above, $n=4 ; i=1,2,3,4$.

Provided particle coordinates [x1, x2], the maximum number of iterations is 200 times; The number of particles in the particle group is $\mathrm{N}=50$; The maximum velocity of the particles is 1 , the minimum speed is -1 ; Function upper limit is 75 , and the lower limit is -5 .

$$
\begin{aligned}
& t_{i}=\frac{\sqrt{\left(S_{i}(1)-X(1)\right)^{2}+\left(S_{i}(2)-X(2)\right)^{2}}}{c} \\
& d_{i}=\sqrt{\left(S_{i}(1)-x 1\right)^{2}+\left(S_{i}(2)-x 2\right)^{2}} \\
& \mathrm{t}_{0}=\frac{1}{4} \sum_{i=1}^{4}\left(t_{i}-\frac{d_{i}}{c}\right) \\
& \mathrm{F}\left(\mathrm{X}_{m}\right)=\frac{1}{4} \sum_{i=1}^{4}\left(t_{i}-\frac{d_{i}}{c}-\mathrm{t}_{0}\right)^{2}
\end{aligned}
$$

TOA/DF-PSO algorithm. Set parameter as in the part TOA-PSO algorithm.

In addition to the calculation of Eq.9, Eq.10, Eq.11 and Eq.12, but also to give as the follows:

$$
\varphi_{i}=\arctan \frac{y_{i}-X(2)}{x_{i}-X(1)}
$$

$$
\mathrm{F}\left(\mathrm{X}_{m}\right)=\frac{1}{4} \sum_{i=1}^{4}\left(\left(t_{i}-\frac{d_{i}}{c}-\mathrm{t}_{0}\right)^{2}+\left(\varphi_{i}-\arctan \frac{y_{i}-x 2}{x_{i}-X 1}\right)^{2}\right)
$$

TOA-PSO algorithm's results is analyzed and compared with the TOA/DF-PSO algorithm. Lightning-points the two algorithms obtained are as shown in Table 1.

Table 1 TOA-PSO algorithms and TOA/DF algorithm simulation proceeds lightning point

Lightning-points the two algorithms obtained

\begin{tabular}{c|c}
\hline TOA-PSO lightning point (units are $\mathrm{km})$ & TOA/DF-PSO lightning point (units are $\mathrm{km})$ \\
\hline$[25.000000000000000,24.999999999999908]$ & {$[25.000000000000281,25.000000000000068]$} \\
\hline$[24.9999999999999940,24.999999999999989]$ & {$[24.9999999999999485,25.000000000000458]$} \\
\hline$[24.999999999994973,25.000000000001126]$ & {$[24.999999999999002,24.999999999999488]$} \\
\hline$[24.9999999999999311,25.000000000000778]$ & {$[24.999999999999840,24.9999999999999982]$} \\
\hline
\end{tabular}




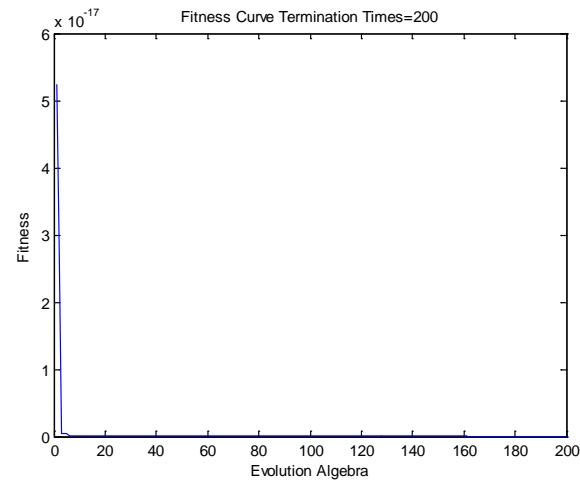

(a) TOA-PSO fitness curve

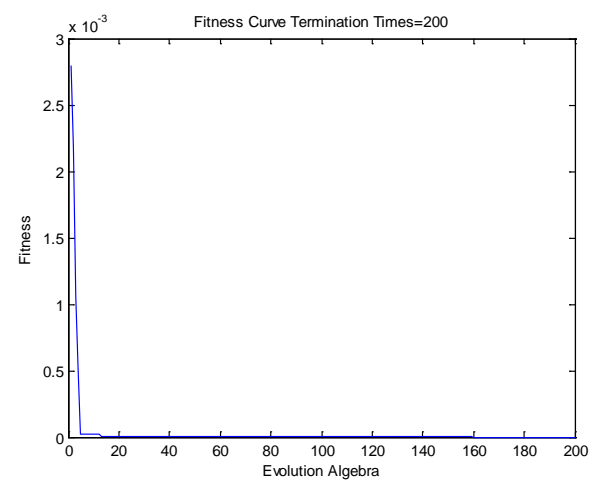

(b) TOA/DF-PSO fitness curve

Figure 2 TOA-PSO algorithm's and TOA/DF algorithm's fitness curve

Table 1 shows that both algorithms have obtained a lightning position (the result is very close to $X[25,25])$.Without considering the measurement errors, the positioning accuracy is quite high. Thus verify the accuracy and feasibility of the two algorithms. From Figure 2, PSO algorithm converges fast and have good convergence.

\section{Summary}

This paper introduced the principle and calculation process of PSO and the numerical simulation analysis. Verified the result that using PSO to achieve lightning location is accuracy and feasibility.

\section{References}

[1] Yi Zhang and Zhang Feng. Zhejiang: Electric Power, Vol.2 (2005), p. 26-29.

[2] Wang Liqun. A new dynamic target detection and tracking technology research [D]. Tianjin University of Technology, 2008.

[3] Zhang Yingying. Non-probabilistic reliability analysis based on Particle Swarm Optimization and Its Application in Slope Engineering [D]. Central South University, 2011

[4] Liu Wei, Chen Hexin, Chen Hanning and so on: Jilin University: Information Science,Vol.29 (2011) , p. 121-127.

[5] Hu Zhixiang. Lightning location algorithm and error analysis theory [D]. Huazhong University of Science and Technology, 2012. 